\title{
The Generation of Data Report of Roller Automatic Production Line Detection Unit
}

\author{
Yuan LIANG ${ }^{1}$, Jian-Ye SUN ${ }^{1, a}$ and Cheng-Feng WEI ${ }^{1}$ \\ ${ }^{1}$ Shenyang Ligong University, Engineering Technology Research Center for High Speed Cutting, \\ Shenyang 110159, China
}

Keywords: WinCC, Data Reporting, Roller automatic production line.

\begin{abstract}
The detecting unit of the roller automatic production line detects the key parameters of each roller and archive them, to analyze whether the roller product performance standards according to the detecting information. The detecting data was stored into SQL database using the VBS of the WinCC software in the host computer. The data in the database were read out to report forms using the VBA in EXCEL for producer to analysis. To solve practical problems and good results have been achieved.
\end{abstract}

\section{Introduction}

Roller is the main transport conveyor parts and wearing parts, its main performance indicators are radial and axial movement, flexibility, and so on. Automatic production line of roller unit, used CCD sensors, torque sensors and other detection devices, real-time current workpiece main performance parameter, and uses the collected data to the PC. Automatic production line of roller monitoring system is to use the powerful Siemens WinCC configuration software[1,2] design and development, WinCC itself with statements and curve-handling capabilities, but for some of the more complex data processing (such as data query, data, statistics, and so on) function appeared to be inadequate. Therefore, database, scripting language and Excel software exploitation reporting system in order to solve the automatic production line of roller unit complex reporting issues in an effective way

\section{Reporting System Design}

This reporting system is mainly used for analysis and treatment of roller production line testing unit of the detected signal, first of all, in the WinCC project, established in various key performance indicators through the variable Manager corresponding external variable, and then by WinCC VBS script language, and to assign the values of external variables in the VBS variables and custom SQL to connect to the database. Custom WinCC6.0 the bottom of the SQL database using database SQL2000, WinCC issued the performance parameters in data will be stored. Excel VBA scripting is an extension of Visual Basic language syntax and VB is very close. VBA can be used to custom SQL database access language, but also through specific program code to bring the data to filter, graphics, tables, and other forms to show up. Report system flow diagram in Figure 1.

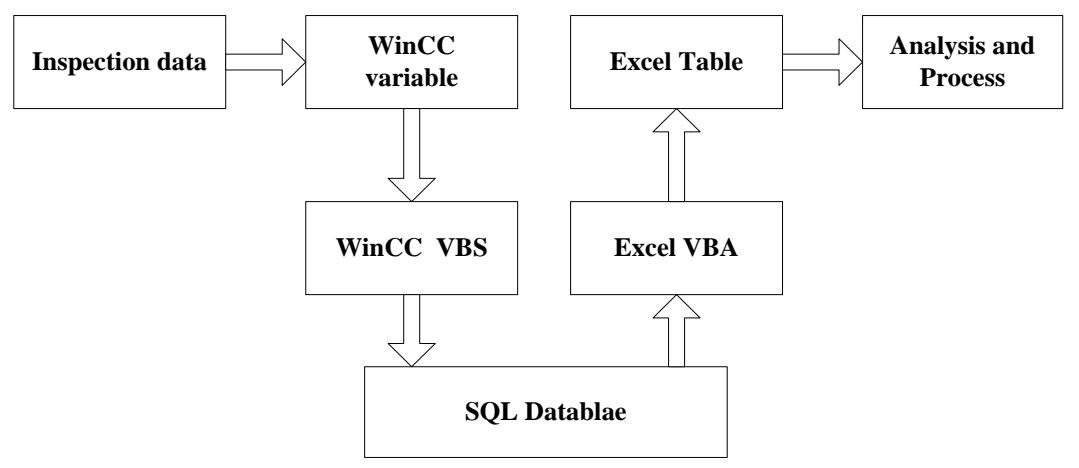

Figure 1. Report system information flow 


\section{Created WinCC External Variables}

According to the detecting unit detects several data characteristics, respectively the corresponding external variable. Nine 32-bit floating point type of an external variable and one binary Boolean variables were established. In the detection unit, each finished testing an artifact, the "MesSave" variable values change, be detected in order to monitor the number of artifacts, in addition to the variables in the following VBS also global variables in the script trigger.

\section{Create SQL Database, and Data Table}

Enterprise Manager using SQL database, establishing a database of "TGodbc02", then users of the database can be set according to user needs access permissions, user name and password, or you can select the default. Data tables in the database are used to actually store the data entities, in setting up a data table, data form must be determined according to the actual situation of the format, content, data and other information. As shown in Figure 2, based on WinCC projects $[3,4]$ have been built in several external variables in custom "MesData" enter the field name in the data table and the data type, length, and nullability information.

\begin{tabular}{|c|c|c|c|c|c|}
\hline \multirow{3}{*}{$\begin{array}{l}\text { TGodbc02 } \\
\text { 嘪 Diagrams } \\
\text { Tables } \\
60 \text { Views }\end{array}$} & \multicolumn{5}{|c|}{ idi Design Table 'IesData' in 'IGodbc02' } \\
\hline & & Column Hame & Data Type & Length & llow Hull \\
\hline & 8 & TGtime & datetime & 8 & \\
\hline Stored Pro & & Er1 & real & 4 & $\checkmark$ \\
\hline Q Users & $D$ & Er2 & real & 4 & $\checkmark$ \\
\hline 6 Roles & & $T_{\min }$ & real & 4 & $\checkmark$ \\
\hline Rules & & F1min & real & 4 & $\checkmark$ \\
\hline Defaults & & E11 & real & 4 & $\checkmark$ \\
\hline User Defin & & E12 & real & 4 & $\checkmark$ \\
\hline $\begin{array}{l}\Sigma_{A c} \text { User Detin } \\
T G Q T\end{array}$ & & E13 & real & 4 & $\checkmark$ \\
\hline 盟 & & F2min & real & 4 & $\checkmark$ \\
\hline Tables & & F3min & real & 4 & $\checkmark$ \\
\hline $60^{\prime} V$ iews & - & GZ & int & 4 & $\checkmark$ \\
\hline
\end{tabular}

Figure 2. SQL Database and Table

\section{Data Storage}

Data storage process of achieved is completely through WinCC of global script achieved of, global script of triggered way has variety, according to detection unit reality, since defined database in the storage of data should is each artifacts of eventually of detection results (the parameter most has may exceeded of limit value), so, should used variable triggers of method, using "MesSave" variable of value each change once, VBS global script will run once, achieved on each current artifacts detection data of storage

\section{Using VBS Script Reads the WinCC External Variables}

First, in order to prevent misspelling a variable name and cause unexpected results when you run a script in the global script, use the Option Explicit statement, shown declaring all variables, that is, all variables by using the Dim statement in a VBS script is defined, then HMIRuntime is used. Tags("").Read statements assign a value to WinCC external variable to specify the VBS script variables.Specific procedures are as follows:

Option Explicit

Function action

Dim objConnection

Dim strConnectionString

Dim lngValue1

Dim lngValue9 
Dim strSQL

Dim objCommand

If HMIRuntime. Tags ("MesSave").Read=1 Then

lngValue1=HMIRuntime. Tags ("Radius1").Read

......

'IngValue9=HMIRuntime. Tags ("Pressure").Read

Else

End If

End Function

In Program, If ...Then...Else statement is used, and real-time judgment variable "MesSave" values, which fires after the program runs.

\section{Write Data to a Custom Database}

\section{Connect to the Database}

There are many ways of connecting to a database, you can use ODBC, OLEDB, ADO, etc, this is the way ODBC using WinCC VBS script to custom SQL database access code. ODBC (Open DataBase Connectivity open database connectivity) is a standard interface for accessing databases, applications to access a database, you must first add a custom ODBC data manager data source, data source provides the data manager database name, database type, ODBC drivers, and information, establish ODBC specific database links, so that The data source will become a registered data source, thereby making the data in the database with a better opening. Custom data source as shown in Figure 3.

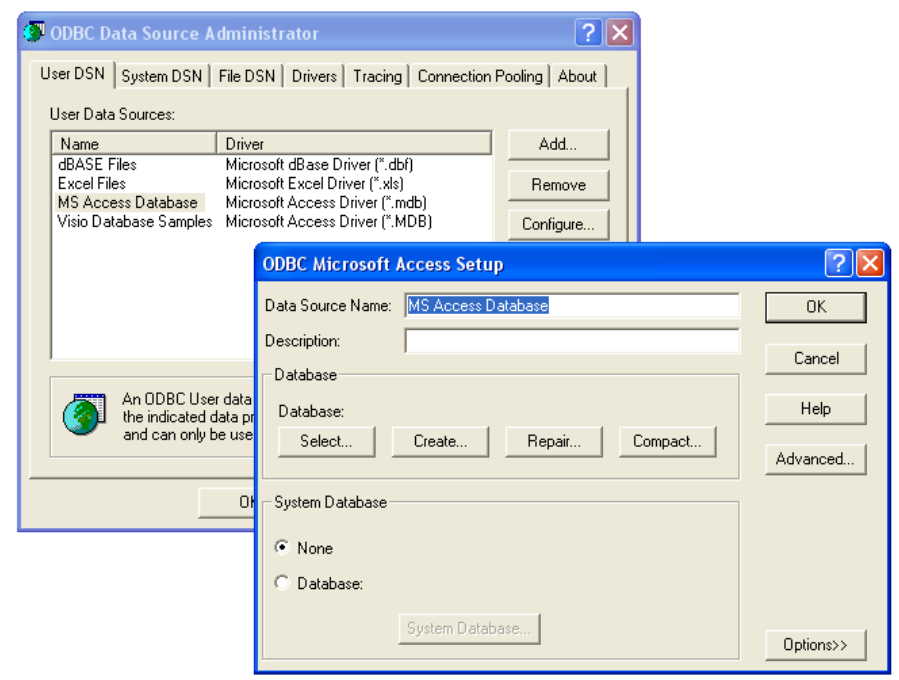

Figure 3. Data Source

\section{Write Database Data}

Written to the database through the standard connection program, due to the ODBC data source supports SQL statements, so in VBS can be achieved through SQL statements in custom database operations. Main program statement:

Connect data source:

strConnectionString="Provider=MSDASQL;DSN=TGodbc02;UID=;PWD=;"

SQL:

strSQL="INSERT INTO MesData(TGtime,Er1,Er2,Tmin,F1min,El1,El2,El3,F2min,F3min) VALUES ('" \& Now()\& "',"'\& lngValue1 \& "','"\& lngValue2 \& "','"\& lngValue3 \& "','"\& lngValue4 \& "','"\& lngValue5 \& "','"\& lngValue6 \& "',"'\& lngValue7 \& "',"'\& lngValue8 \& "',"'\& lngValue9 \& "');" 
Connection properties and statements and execute SQL statements:

Set objConnection=CreateObject ("ADODB. Connection")

objConnection. ConnectionString=strConnectionString

objConnection.Open

Set objCommand=CreateObject ("ADODB. Command")

With objCommand

.ActiveConnection=objConnection

.CommandText=strSQL

End With

objCommand.Execute

Closes the connection and releases the memory space:

Set objConnection $=$ Nothing

Set objCommand=Nothing

objConnection.Close

Set objConnection=Nothing

\section{Excel Report Builder}

\section{Reports Management}

Report feature is designed to facilitate staff to manage the generated data, so it should be possible to use Excel VBA scripting features more reporting features, should have conditions of storage time display, data queries, automatically-generated graphics, and standard features such as data highlighted. Report template as Figure 4 by shows, dang click "query began" button Shi, report system will automatically pop-up conditions query window, entered need query of time paragraph Hou, click "query" button, system will will generated report data and pictures, while also on by generated of data for part operation and filter, for example for individual exceeded of parameter items, font will automatically variable red, corresponds to of fault code will displayed exceeded items of location.

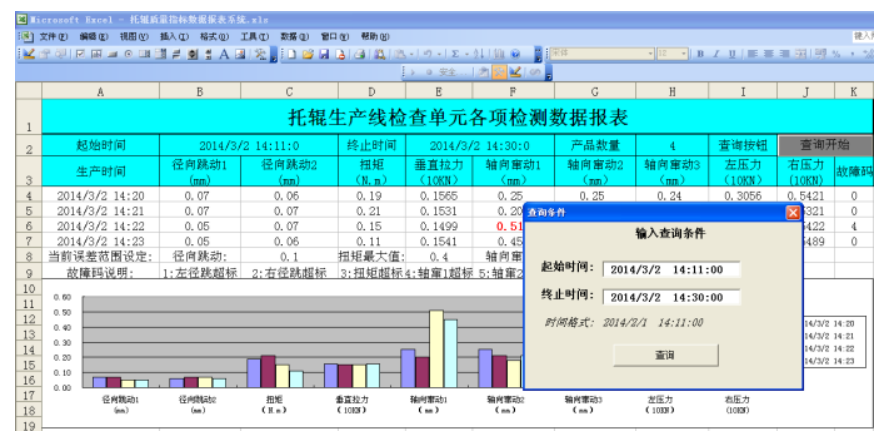

Figure 4. Report template

\section{Report Implementation of the Main Functions}

Report functions mainly through Excel VBA scripting program. By VBA script to access custom data in a database is a key step in Report Builder, because VBA and VB and VBS language is very similar to using ODBC connection "TGodbc02" database procedure is basically the same, program flow chart as shown in Figure 5. 


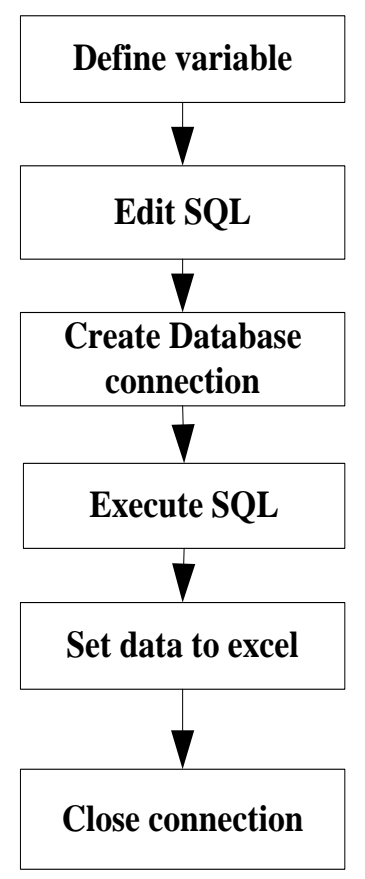

Figure 5. Program flow chart

Connect to the database and assign a value to a cell in the main program code: strConnectionString="Provider=MSDASQL;DSN= TGodbc02;UID=;PWD=;" Set objConnection=CreateObject("ADODB.Connection")

Set objRS = CreateObject("ADODB.Recordset")

Set objCommand $=$ CreateObject("ADODB.Command")

objConnection.ConnectionString $=$ strConnectionString

objConnection. CursorLocation $=3$

objConnection.Open

With objCommand

.ActiveConnection $=$ objConnection

.CommandText $=$ strSQL

End With

Set objRS $=$ objCommand.Execute

$\mathrm{m}=$ objRS.RecordCount ' Total number of query record sets

Sheet1.Cells $(2,2)=\operatorname{strTime} 1$

Sheet1.Cells $(2,5)=$ strTime2

Sheet1.Cells $(2,8)=m$

If Not (objRS.EOF And objRS.bof) Then

objRS.MoveFirst ' Pointer to the first message database

For $\mathrm{i}=4$ To $\mathrm{m}+3$

Sheet1.Cells $(i, 1)=$ objRS.Fields $(0)$.Value 'objRS.Fields $(0)$

Sheet1.Cells $(i, 2)=$ objRS.Fields $(1)$.Value

Sheet1.Cells $(i, 11)=$ objRS.Fields $(10)$.Value

objRS.MoveNext ' Move the pointer to the current database for the next

Next i

Sheet1.Cells $(m+4,1)=$ " Current range setting:"

Sheet1.Cells $(m+5,7)=$ "6:"

Else

End If 


\section{Conclusion}

For solution supporting roll Automation line detection Unit report generated of problem, to supporting roll line monitoring system of upper machine for platform, used WinCC since with of VBS global script achieved has will detection data deposit to since defined of SQL database in the, then used Excel of VBA script achieved has on since defined database of conditions query, while, using VBS script achieved Excel of other management function, improve has report system data processing of flexibility. Design data reporting system has been applied in roller production line, with good results.

\section{References}

1. Implementation of WinCC data report [k].Siemens official website

2. J.M. Guo, C. Xing, Q. Liu. WinCC configuration software realization of customized complex reports. Industrial control computer, 20 (2), 1-2 (2007).

3. L. Liu, X.G. Yang. QteCt improvement and application of the industrial control configuration software reporting features. Industrial control computer, 14(4): 44- 46 (2001).

4. H.B. Liu, S. King, W.X. He. Configuration software WinCC and its application. Machinery. X21k Publishing House (2009). 